\title{
Ensuring Bi-Directional Cavo-Tricuspid Isthmus Conduction Block During Ablation for Typical Atrial Flutter - A New Twist on an Old Problem
}

\author{
Gregory Feld ${ }^{1}$ \\ ${ }^{1}$ University of California-San Diego
}

May 6, 2020

\begin{abstract}
This editorial will review the new pacing method described by the authors in this issue of the journal that they propose to ensure bi-directional cavo-tricuspid isthmus (CTI) conduction block after radiofrequency catheter ablation in patients with CTI dependent atrial flutter.

Ensuring Bi-Directional Cavo-Tricuspid Isthmus Block During Ablation for Typical Atrial Flutter - A New Twist on an Old Problem

Gregory K. Feld, MD

Professor of Medicine

Depart of Medicine, Division of Cardiology

Director, Clinical Cardiac Electrophysiology Program

and CCEP Fellowship Training Program

University of California San Diego Health System

Address for Correspondence:

Gregory K. Feld, MD

9452 Medical Center Drive

Altman CTRI Building

$3^{\text {rd }}$ Floor, Room 3E-313

La Jolla, CA 92037

Although it has been nearly 20 years since the first descriptions of the methods for radiofrequency ablation (RFA) of the cavo-tricuspid isthmus (CTI) for treatment of typical and reverse typical atrial flutter (AFL $)^{1,2}$, and nearly 15 years since the first description of methods to ensure bi-directional CTI conduction block to improve efficacy of RFA for cure of $\mathrm{AFL}^{3,4}$, this paper by Jiménez-López, et.al., in the current issue of Journal of Cardiovascular Electrophysiology ${ }^{5}$, provides subtle additional methods to ensure that bidirectional CTI conduction block is achieved during RFA in order to further reduce recurrence rates of this common arrhythmia.
\end{abstract}


The limitations of current methods for determining CTI conduction block (e.g. demonstration of a fully descending limb of electrical activation in the contralateral atrial wall during pacing in sinus rhythm after ablation $^{5}$, a trans-isthmus conduction time measured as a double potential interval along the ablation line $>100-110$ milliseconds $(\mathrm{msec})^{5}$, responses to differential and incremental pacing maneuvers associated with apparent CTI conduction block including changes in activation times and electrogram morphology ${ }^{6.7}$ ) are identified and compared to the novel incremental pacing (IP) maneuver measurements proposed by the authors in this paper.

Specifically, in this issue of the journal ${ }^{5}$, the authors propose a new method for measuring conduction times across and adjacent to the CTI ablation line during IP, which are independent of other anatomical determinants of conduction delays in the right atrium that may be due to other factors (e.g. atrial scarring). In addition, this new method of measurement allows for assessment of bi-directional conduction across the CTI, whereas some other formerly accepted methods only described uni-directional conduction assessment during $\mathrm{IP}^{, 5}$.

Briefly, this novel or "variant IP maneuver" proposed by the authors involves IP from the low lateral right atrium and the coronary sinus at progressively shorter cycle lengths from 600 to $300 \mathrm{msec}$, while measuring the activation time between two electrodes, one placed in close proximity $(<5 \mathrm{~mm})$ to the ablation line and one adjacent to that but on the contralateral side from the pacing site. Using this approach, the authors hypothesized that after initial CTI ablation a $>10$-ms increase in the time interval between the low lateral right atrium and an adjacent site immediately contiguous $(<5 \mathrm{~mm})$ to the lateral aspect of the CTI line during IP from the coronary sinus ostium would indicate persistent clockwise CTI conduction, and vice versa with respect to the proximal coronary sinus electrogram timing relative to the activation timing of an electrogram just medial to the CTI line during pacing from the low lateral right atrium. Furthermore, the authors hypothesized that their IP variant maneuver would be more accurate in confirming bidirectional CTI conduction block and uncovering functional CTI conduction block or conduction delay (even in the presence of right inter-atrial conduction delay) compared to conventional IP maneuvers or the so-call His-tocoronary sinus IP maneuver, thus potentially ensuring adequate ablation in some cases, reducing the amount of ablation needed in others, and resulting in better long-term outcomes in patients undergoing ablation for CTI dependent atrial flutter.

From the data reported by the authors it appears that about $14 \%$ (15 of 108) of their patients had fractionated or indeterminate electrograms recorded on or near the CTI ablation line making measurements during IP maneuvers impossible, and thus these patients were excluded from the analysis. This is not an insignificant number of patients in whom this type of analysis cannot be performed, and in this author's observations, rapid atrial pacing to demonstrate persistent inducibility of typical or reverse typical AFL, and observing atrial activation patterns during pacing maneuvers with 3-dimensional electro-anatomical mapping (3-D EAM) when being used, may help to identify patients in this group that cannot be evaluated with conventional or variant IP maneuvers or those with false positive findings suggesting complete CTI conduction block. The authors of this paper don't reiterate the importance of ruling out persistently inducible atrial flutter in all their patients after CTI ablation, but it is likely that they do so, as well as perform the described pacing maneuvers to ensure bi-directional CTI conduction block, but this might have an influence on long-term outcomes as well. In addition, there is no mention of the use of 3-dimensional mapping in their patients, but it has also been this author's observation that 3-D EAM may identify small gaps in the CTI ablation line with functional block or slow conduction, that allows persistently inducible AFL and late AFL recurrence.

Among those patients in whom this variant IP maneuver could be performed, the authors found that their novel method increased the number and percentage of patients in whom persistent functional conduction block or conduction delay could be identified in comparison to the conventional IP maneuver (e.g. from 69 [74\%] using the gold-standard conventional IP maneuver to 65 [68\%] using the novel IP maneuver), resulting in additional ablation being performed along the ablation line. But, there was apparently no difference when compared to the conventional His-to-coronary sinus IP maneuver.

Another potentially important finding from the prospective arm of this study in patients who also had right 
inter-atrial conduction delay, is that the author's novel IP maneuver allowed reclassification of 7 of 11 (64\%) patients from a functional to complete block status, thus preventing the need for additional ablation that might have otherwise been deemed necessary if only the conventional IP maneuvers were used to assess CTI conduction. This might be considered a safety benefit by some.

The authors also included in their study, an evaluation of the effect of this novel IP maneuver in comparison to conventional IP maneuvers on the long-term recurrence rates of AFL. Although the numbers of patients with AFL recurrence in this study is very small, and this observation would have to be evaluated and reproduced in a much larger population of patients, the findings were nonetheless statistically significant with respect to the use of this novel IP maneuver compared to the conventional IP maneuver studied $(p=0.1)$. This observation can be seen in the negative and positive predictive values presented by the authors for the conventional IP maneuver, the standard His-to-coronary sinus IP maneuver and their novel IP maneuver (i.e. 50\%, 75\% and $100 \%$ negative prediction value and $97 \%, 98 \%$ and $100 \%$ positive predictive value, respectively).

Furthermore, with regards to the follow-up of patients in this study to assess for recurrence of AFL, the authors note in their limitations section of the paper that the study might be negatively impacted by the small numbers (as mentioned above), but also by the lack of use of an internal loop recorder. While the use of such monitoring may help identify a higher percentage of patients with arrhythmia recurrence, especially asymptomatic recurrences, this technique may not be employed in many studies or even required by some regulatory agencies (e.g. in some cases only an extended monitor such as a 48-hour Holter is required during follow-up). In this study a 24-hour Holter monitor was only applied when "relapses were suspected", suggesting that it was applied only in case of symptomatic recurrence. This lack of systematic use of a Holter monitor (or extended event monitor) or implantable loop recorder ${ }^{8}$ may have indeed led in part to the small number of recurrences of AFL observed in this study, since reported recurrence rates of AFL are usually closer to $10 \%$ in most studies after CTI ablation ${ }^{9}$. Thus, both a much larger study and the routine use of extended monitoring may have resulted in more reliable data to support the authors' contention that use of this novel IP maneuver improves long-term outcomes with regards to recurrence of AFL in patients undergoing CTI ablation.

In summary, this study adds a small but useful tool to our armamentarium for assessment of bi-directional conduction block in patients undergoing RF ablation for CTI dependent AFL. The greater ability of this novel IP maneuver, compared to conventional IP maneuvers, to distinguish complete CTI conduction block from functional block or conduction delay, may help reduce unnecessary ablation by confirming the presence of complete conduction block when other methods suggest only incomplete block, and may also improve longterm outcomes after RF ablation for CTI dependent AFL by confirming the presence of complete conduction block which is known to be necessary to prevent AFL recurrence (again with the caveat that larger studies with more robust methods of detecting recurrences may be necessary to confirm this latter suggestion).

\section{References}

1. Feld GK, Fleck RP, Chen PS, Boyce K, Bahnson TD, Stein JB,Calisi CM, Ibarra M. Radiofrequency catheter ablation for the treatment of human type 1 atrial flutter: Identification of a critical zone in the re-entrant circuit by endocardial mapping techniques. Circulation 86:1233-1240,1992.

2. Cosio FG, López-Gil M, Goicolea A, Arribas F, Barroso JL Am J Cardiol. Radiofrequency ablation of the inferior vena cava-tricuspid valve isthmus in common atrial flutter. 1993:71:705-9. Am J Cardiol.

3. Poty H, Saoudi N, Aziz AA, Nair M, Letac B. Radiofrequency catheter ablation of type I atrial flutter. Prediction of late success by electrophysiological criteria. Circulation 1995;92:1389-1392.

4. Schwartzman D, Callans DJ, Gottlieb CD, Dillon SM, Movsowitz C, Marchlinski FE. Conduction block in the inferior vena caval-tricuspid valve isthmus: Association with outcome of radiofrequency ablation of type I atrial flutter. J Am Coll Cardiol 1996;28:1519-1531.

5. Jiménez-López J, Vallès E, Martí-Almor J, González-Matos C, Bas D, Benito B, Alcalde O, Cabrera S, Altaba C, Bazan V. Mapping Potentials Adjacent to the Cavo-tricuspid Isthmus Ablation Line during Incremental Pacing: A Feasible and Highly Accurate Maneuver to Confirm Complete CTI Conduction Block. J Cardiovasc Electrophysiol 2020:??;???. 
6. Vallès E, Bazán V, Benito B, Jáuregui ME, Bruguera J, Guijo MA, Altaba C, Martí-Almor J. Incremental His-to-coronary sinus maneuver: a nonlocal electrogram-based technique to assess complete cavotricuspid isthmus block during typical flutter ablation. Circ Arrhythm Electrophysiol 2013;6:784-9.

7. Shah D, Haissaguerre M, Takahashi A, Jais P, Hocini M, Clemency J. Differential pacing for distinguishing block from persistent conduction through an ablation line. Circulation 2000;102:1517-1522.

8. Giada F, Gulizia M, Francese M, Croci F, Santangelo L, Santomauro M, Occhetta E, Menozzi C, Raviele A. Recurrent unexplained palpitations (RUP) study comparison of implantable loop recorder versus conventional diagnostic strategy. J Am Coll Cardiol 2007;49:1951-6.

9. Pérez FJ, Schubert CM, Parvez B, Pathak V, Ellenbogen KA, Wood MA. Long-term outcomes after catheter ablation of cavo-tricuspid isthmus dependent atrial flutter: a meta-analysis. Circ Arrhythm Electrophysiol. 2009;2:393-401. 